\title{
Annual Exhibition of the Royal Photographic Society
}

7 HE seventy-eighth Annual Exhibition of the Royal Photographic Society is being held at the Society's rooms, 35, Russell Square, W.C.1, from September 9 until October 7. Lectures are given on Tuesday and Friday evenings during that period.

As in former years, there is much to be seen that is interesting from a scientific, as well as a photographic, point of view. The deciphering of documents by infra-red and ultra-violet radiation is illustrated, and there is one example in which success has been achieved by leaving a charred document in contact with a photographic plate for forty days. There is a remarkable set of photomicrographs of diffraction gratings, the spacings of the grooves being 300,600 and 900 per $\mathrm{mm}$.

Perhaps the most outstanding exhibits are the astronomical and spectrum prints from the Mount Wilson Observatory. Among these are five spectroheliograms of the eruptive solar prominence of August 6, 1931, taken at half-hour intervals. There is also a print showing four faint nebulæ at distances ranging from $23,000,000$ to $105,000,000$ light years, together with the spectrum of each. Another shows a display of stellar spectra with dispersion increasing from $835 \mathrm{~A}$. per $\mathrm{mm}$. to so much as $0.7 \mathrm{~A}$. per $\mathrm{mm}$. Of the series, the most notable is the spectrum of Jupiter, a comprehensive piece of work from the same source showing the presence of ammonia in the planet's atmosphere. For this the Society has awarded a medal. Included are spectra of the sun and of Mars taken while the planet was approaching and receding from the earth. These show displacement of the solar lines relative to the oxygen band and indicate that in the atmosphere of Mars there cannot be an amount of oxygen exceeding 1 per cent of that in our own atmosphere.

By means of an electric spark, photographs have been taken in $1 / 75,000$ sec. at $F 16$ of an electric light bulb falling to pieces under the stroke of a hammer. In the same series is a print of a cup of coffee striking the floor and one of water apparently arrested in the act of flowing into a milk bottle.

An exhibit has been staged to show that the graininess of photographic material normally increases with its speed and that this is mainly due to the fact that fast emulsions contain large-sized crystals of silver bromide. The usual means at the disposal of the photographer for combating graininess are indicated and the real solution of the problem is shown to lie with the emulsion maker. That it is not an insuperable problem is demonstrated by comparison pictures showing the successful diminution of grain in new negative materials as compared with old without sacrifice of speed or quality.

Another feature of interest is a new method of optical intensification in which the image is converted to silver bromide and then sulphided in one of three types of bath each of which gives an image of distinctive qualities. Intensification takes place by exposure under solutions of sodium silver nitrite or sodium silver sulphite to a 100 watt or 200 watt lamp.

Classed under "Technical Applications" are pictures transmitted by telegraphy using the Siemens. Karolus-Telefunken system. These are from the Picture Telegraph Department of the G.P.O.

In the sphere of colour photography there are some excellent examples of results obtained with the Vivex process, in which the pigment image is obtained by chemical contact of a bromide print with carbon tissue. Another exhibit is arranged to show the possibility of eliminating, by photographic means, the need for fine etching in the reproduction of colour with printing inks.

In other sections the usual high standard is maintained. Among the accessories and apparatus on view are two cameras which played important parts in the recent Mount Everest Expeditions-the 'Williamson Eagle' which was used on the flight and the 'Leica' with which photographs were taken by the climbers at $28,000 \mathrm{ft}$.

Demonstrations are given of the results obtained with a new $16 \mathrm{~mm}$. cine-camera with which it is possible for an amateur to produce most of the effects, like 'dissolves' and 'double exposures', which are usually looked upon as beyond his power. It is understood that a $16 \mathrm{~mm}$. sound film is being incorporated in the exhibition.

$$
\text { J. M. }
$$

\section{Wireless Direction of Aircraft Landing in Fog}

$\mathrm{F}^{\mathrm{o}}$ R many years past the application of wireless communication as an aid to air navigation has been studied intensively in various parts of the world. In the United States particular attention has been paid to the development of the course indicator system, by means of which an aeroplane can be navigated along prescribed air routes solely by the indications of wireless signals received from fixed transmitting beacons erected on the ground at appropriate points. By proceeding along a chain of such beacons, it is possible for the air navigator to fly from one aerodrome to another in a dense fog when visual observations become impossible. At a later stage, experiments were carried out in the United States on a means of enabling a pilot to land on the ground in a fog on arrival at the aerodrome.

A description of the mode of achieving this, with some details of experimental 'blind' landings made by aid of the invention, was published in $1930^{1}$. The method makes use of a concentrated beam of ultra. short waves into the line of which the aircraft is directed by the ordinary beacons. On picking up the signals from the beam, which is elevated slightly above the horizontal, the pilot adjusts his receiver until an output meter gives a deflection up to a prescribed mark. From then onwards, the pilot operates his machine in such a manner as to keep this deflection constant. In this way the aircraft 'slides' down the landing beam and reaches the earth tangentially, and the pilot is thus enabled to make a good landing without having seen the ground beneath him.

In the issue of the Wireless World for August 25, a New York correspondent gives a description of the recent installation of this landing beam system at Newark, the largest air port in the United States. 